\title{
Analysis of endometrial blood flow with color Doppler energy in predicting outcome in GnRH antagonist down regulated ICSI/IVF cycles: a prospective cohort study setting
}

\author{
Rai R. ${ }^{1 *}$, Ling Y.S. ${ }^{2}$ Yong T. T. ${ }^{2}$, Keat A. T. ${ }^{2}$, Rajesh H. ${ }^{2}$
}

\begin{abstract}
${ }^{1}$ Department of Obstetrics and Gynaecology, B. P. Koirala Institute of Health Sciences, Dharan, Nepal
${ }^{2}$ Department of Obstetrics and Gynaecology, Singapore General Hospital, Singapore
\end{abstract}

Received: 03 August 2017

Accepted: 01 September 2017

*Correspondence:

Dr. Rai R.,

E-mail: rairubina@yahoo.com

Copyright: (C) the author(s), publisher and licensee Medip Academy. This is an open-access article distributed under the terms of the Creative Commons Attribution Non-Commercial License, which permits unrestricted non-commercial use, distribution, and reproduction in any medium, provided the original work is properly cited.

\section{ABSTRACT}

Background: The study was conducted to assess the association between endometrial blood flow pattern assessed with colour Doppler around the day of HCG administration and IVF outcome following GnRH antagonist down regulated cycles.

Methods: It was a prospective, cohort study. Total of sixty-eight patients undergoing IVF-ET/ICSI were recruited in the study. All the patients underwent controlled ovarian stimulation with a step-up protocol, and GnRH antagonists were used for down-regulation. Transvaginal ultrasound measurements of all patients were performed on the day of HCG injection. A $6.5 \mathrm{MHz}$ pulsed Doppler system was used for blood flow analysis. Bilateral uterine arteries, pulsatility index and resistance index were calculated along with uterine artery peak systolic velocity on both sides. Endometrial blood flow was analysed by detecting flow in the intra-endometrial or the adjacent sub-endometrial regions.

Results: Baseline FSH in pregnant group was lower (6.29) than non-pregnant group (7.36). The overall pregnancy rate was $45.6 \%(n=31)$ and the ongoing pregnancy rate was $41.1 \%(n=28)$. Total of 57 patients out of 68 patients had both good endometrial and sub-endometrial blood flow. The overall pregnancy rate in this group was $47.3 \%$. Similarly, in patients who had minimal endometrial and sub-endometrial blood flow the pregnancy rate was $37.5 \%$. There was no significant correlation between pregnancy outcomes and the color flow Doppler parameters such as average uterine PI, average uterine RI and right/left uterine peak systolic velocity.

Conclusion: Uterine artery PI, RI and PSV has no role in predicting endometrial receptivity and thus pregnancy outcome in IVF-ET cycle, however those patient with good endometrial and sub-endometrial flow have higher pregnancy rate than those with minimal flow rate.

Keywords: Doppler study, Endometrial blood flow, IVF

\section{INTRODUCTION}

In Vitro Fertilization involves a sequence of highly coordinated steps beginning with controlled ovarian hyperstimulation with exogenous gonadotropins, followed by retrieval of oocytes from the ovaries under the guidance of transvaginal ultrasonography, fertilization in the laboratory and trans-cervical transfer of embryos into the uterus. Gonadotropin-releasing hormone (GnRH) antagonist is now widely used in controlled ovarian stimulation cycles, with advantages of shorter duration of treatment than agonist for pituitary down regulation, decrease requirement of total dose and duration of gonadotropin stimulation and has lower risk for ovarian hyperstimulation syndrome. ${ }^{1}$ Maternal Age, quality of the embryo and endometrial receptivity are the most 
important factors for the success of IVF. Sonographic evaluation of Endometrial thickness (EMT) has been accepted as an indicator for endometrial receptivity, and assessment of the endometrium in the midsagittal plane via transvaginal ultrasound is the standard procedure. More recently endometrial blood flow assessment with colour Doppler energy has been used in predicting pregnancy outcome in IVF-ET cycles. ${ }^{2}$ Several studies have been conducted to evaluate the effect of controlled ovarian hyperstimulation with GnRH agonist and antagonist on endometrium receptivity or implantation with conflicting results. ${ }^{3-8}$ Moreover, GnRH antagonist for pituitary down regulation during ovulation induction has been seen to directly act on extra pituitary receptor, including the endometrium and has been associated with decrease endometrial receptivity. ${ }^{9}$ There are few studies examining the association between endometrial receptivity measured in the form of endometrial blood flow with colour Doppler and clinical outcome in the GnRH antagonist protocol. ${ }^{2,10}$ The aim of this study was to assess the association between endometrial blood flow pattern assessed with colour Doppler around the day of HCG administration and IVF outcome following GnRH antagonist down regulated cycles.

\section{METHODS}

This study was a prospective study undertaken at the CARE (Centre for Assisted Reproduction) Unit of department of Obstetrics and Gynecology, Singapore General Hospital, Singapore and was approved by the Institutional Review Board. Informed and written consent were obtained from the women willing to participate in the study. Total of sixty-eight patients undergoing IVFET/ICSI were recruited in the study. All the patients underwent controlled ovarian stimulation with a step-up protocol, and GnRH antagonists were used for downregulation. Recombinant FSH was administered from day 2 or day 3 of menstruation until the day of the HCG trigger for ovarian stimulation. GnRH antagonist was added from the day when at least one follicle reached 12 $\mathrm{mm}$ in diameter and continued until the day of the HCG trigger.

Transvaginal ultrasound measurements of all patients were performed on the day of HCG injection after they had emptied their bladder. A 6.5 MHz pulsed Doppler system was used for blood flow analysis. Bilateral uterine arteries Pulsatility index and resistance index were calculated along with uterine artery peak systolic velocity on both sides. Endometrial blood flow was analyzed by detecting flow in the intra-endometrial or the adjacent sub-endometrial regions within $10 \mathrm{~mm}$ of the echogenic endometrial borders. According to the endometrial blood flow the patients were divided into 4 groups.

- Group 1: Good endometrial and sub-endometrial blood flow detected

- Group 2: Minimal endometrial and sub-endometrial blood flow detected
- Group 3: Endometrial blood flow present and subendometrial blood flow absent

- Group 4: Endometrial flow absent and subendometrial flow present.

Patients had day 3, two embryos of reasonable quality replaced into the uterine cavity 48 to 72 hours after the oocyte retrieval. Luteal phase support was given in the form of oral dydrogestrone tablet $10 \mathrm{mg}$ three times a day. The patients were followed up for the outcomes. Serum beta HCG was performed on day 14 after embryo transfer. Ultrasound examination was performed 4 weeks after embryo transfer to confirm intrauterine pregnancy. The patients were divided into three groups.

\section{- Group 1: Non-pregnant}

- Group 2: Intrauterine pregnancy with live fetus

- Group 3: Pregnancy with poor outcome including ectopic, biochemical and miscarriage.

\section{Statistical analysis}

Statistical Package for Social Sciences (SPSS) 11.5 software was used to analyze the data. Continuous data are presented as mean \pm SD. Mean were compared between pregnant and non-pregnant group using independent sample mean comparison test at $95 \% \mathrm{CI}$ where $\mathrm{p}$ value $=0.05$. For categorical data chi-square test was used to find significant difference between pregnant and non-pregnant group with endometrial and endometrial flow rates.

\section{RESULTS}

During the study period, a total of 68 patients receiving gonadotropin antagonist down regulated cycles were enrolled for the study. Mean age group was 34 years. Baseline FSH in pregnant group was lower (6.29) than non-pregnant group (7.36). This difference in mean FSH between the two groups was statistically significant $(\mathrm{P}=0.016)$. However, there was no statistically significant difference between the AFC, Day 2 Estradiol, E2 at the day of HCG and progesterone level at the day of HCG injection between the pregnant and non-pregnant group (Table 1).

In the four groups divided according to the pattern of endometrial and sub-endometrial blood flow imaging, Group 1, Group 2, Group 3 and Group 4. Total of 57 patients out of 68 patients had both good endometrial and sub-endometrial blood flow and came under Group 1. The overall pregnancy rate in this group was $47.3 \%$. Similarly, in Group 2 who had minimal endometrial and sub-endometrial blood flow the pregnancy rate was $37.5 \%$. The sample sizes in other 2 groups were very small to make any inferences. The overall pregnancy rate was $45.6 \%(n=31)$ and the ongoing pregnancy rate was $41.1 \%(\mathrm{n}=28)$. 
This study did not find any relationship between pregnancy outcomes and the color flow Doppler parameters such as average uterine PI, average uterine RI and right/left uterine peak systolic velocity (Table 2).

Table 1: Comparison of demographic data and ovarian responses between pregnant and non-pregnant group.

\begin{tabular}{|llll|}
\hline Variable & $\begin{array}{l}\text { Non-pregnant }(\mathrm{n}=\mathbf{3 7}) \\
\text { Mean } \pm \text { SD }\end{array}$ & Pregnant $(\mathrm{n}=\mathbf{3 1})$ & p values \\
\hline Age & $35.14 \pm 3.85$ & $34.94 \pm 2.93$ & 0.814 \\
\hline AFC & $22.38 \pm 12.23$ & $23.9 \pm 12.22$ & 0.610 \\
\hline FSH & $7.36 \pm 1.95$ & $6.297 \pm 1.52$ & 0.016 \\
\hline Estradiol D2 & $121.52 \pm 41.16$ & $122.03 \pm 56.13$ & 0.996 \\
\hline E2 at the day of HCG & $8198.35 \pm 3870.83$ & $9498.10 \pm 4797.54$ & 0.221 \\
\hline P4 at the day of HCG & $2.74 \pm 0.83$ & $2.81 \pm 0.93$ & 0.749 \\
\hline
\end{tabular}

Table 2: Relationship between color Doppler imaging parameters and IVF-ET outcomes.

\begin{tabular}{|llll|}
\hline Doppler parameters & Non-pregnant $(n=37)$ & Pregnant $(n=31)$ & p values \\
\hline Average uterine PI & Mean \pm SD & $2.55 \pm 0.68$ & 0.113 \\
\hline Average uterine RI & $2.82 \pm 0.69$ & $0.83 \pm 0.60$ & 0.135 \\
\hline Right uterine PSV & $0.86 \pm 0.07$ & $30.52 \pm 13.03$ & 0.604 \\
\hline Left uterine PSV & $32.28 \pm 14.5$ & $35.79 \pm 16.32$ & 0.802 \\
\hline
\end{tabular}

\section{DISCUSSION}

Endometrial receptivity is vital for successful implantation of an embryo. Different strategies have been evaluated to assess the endometrial receptivity including histologic dating of an endometrium, endometrial cytokines in uterine flushing, microarray technology for identification of transcriptomic signature of the window of implantation and more commonly a non-invasive ultrasound examination of the endometrium. ${ }^{11,12}$ Adequate perfusion to the endometrium is essential requirement for implantation of the embryo and therefore assessment of endometrial blood flow using color Doppler ultrasound is believed to be useful in assessing endometrial receptivity. ${ }^{13}$

GnRH antagonist are used in the IVF-ET cycles to prevent premature LH surges, with advantages of shorter duration of treatment than agonist cycles, decrease requirement of total dose and duration of gonadotropin stimulation and lower risk of developing ovarian hyperstimulation syndrome. GnRH antagonist has been shown to modify extra pituitary receptors including the endometrium and has been associated with decrease endometrial receptivity. GnRH antagonist downregulated cycles have been also reported to have worse pregnancy outcomes when compared to GnRH agonist, may be due to a substantial down-regulation of LH. ${ }^{14}$ However the results of the studies comparing the effects of $\mathrm{GnRH}$ agonist and antagonist down regulated cycles on the outcome of IVF-ET are conflicting.

The aim of the present study was to evaluate the role of the endometrial and sub-endometrial blood flow measured by 3D power Doppler ultrasound in the prediction of pregnancy during treatment with GnRH antagonist down regulated cycle.

In the present study, we found no association between sub-endometrial, endometrial blood flow, uterine artery Pulsatility index (PI), Resistance Index (RI), Peak Systolic Velocity (PSV) and pregnancy outcome in IVFET cycle and that it could not be used as the only means for predicting endometrial receptivity. There was no significant difference between PI, RI, and PSV of uterine artery between the pregnant and non-pregnant groups. This finding is similar to the study done by Wang L et al, however the present study included patient receiving only antagonist cycle whereas the study done by Wang L et al included patients receiving only long agonist protocol. ${ }^{2}$

Wang $L$ et al found that patients with detected endometrial blood flow had a higher pregnancy and implantation rate than those with only sub-endometrial blood flow detection or those without detected blood flow. In the present study $83.8 \%$ of patients had both good endometrial and sub-endometrial blood flow and came under Group 1.

The overall pregnancy rate in this group was $47.3 \%$. Similarly, in Group 2 who had minimal endometrial and sub-endometrial blood flow the pregnancy rate was $37.5 \%$, these finding shows that the patient with good endometrial and sub-endometrial flow have higher pregnancy rate than those with minimal flow. In the study done by Singh $\mathrm{N}$ et al, the blood flow directly correlated with the outcome of the IVF cycle and concluded that endometrial vascularity has a useful value on the 
implantation rate in IVF cycles. ${ }^{10}$ These findings are in contrast with that of the present study.

\section{CONCLUSION}

In conclusion, the present study shows that uterine artery PI, RI and PSV has no role in predicting endometrial receptivity and thus pregnancy outcome in IVF-ET cycle, however those patient with good endometrial and subendometrial flow have higher pregnancy rate than those with minimal flow rate even though it did not reach statistical significance. However further study with larger sample size is needed to make a definitive conclusion.

\section{Funding: No funding sources}

Conflict of interest: None declared

Ethical approval: The study was approved by the Institutional Ethics Committee

\section{REFERENCES}

1. Kinay T, Tasci Y, Dilbaz S, Cinar O, Demir B, Haberal A. The relationship between endometrial thickness and pregnancy rates in GnRH downregulated ICSI cycles. Gynecol Endocrinol. 2010;26(11):833-7.

2. Wang L, Qiao J, Li R, Zhen X, Liu Z. Role of endometrial blood flow assessment with color Doppler energy in predicting pregnancy outcome of IVF-ET cycles. Reprod Biol Endocrinol. 2010;8:122.

3. Kasius A, Smit JG, Torrance HL, Eijkemans MJ, Mol BW, Opmeer BC, et al. Endometrial thickness and pregnancy rates after IVF: a systematic review and meta-analysis. Hum Reprod Update. 2014;20:530-4.

4. Aydin T, Kara M, Turktekin N. Relationship between endometrial thickness and in vitro fertilization/intracytoplasmicsperm injection outcome. Int J Fertil Steril. 2013;7:29-34.

5. Kumbak B, Erden HF, Tosun S, Akbas H, Ulug U, Bahçeci M. Outcome of assisted reproduction treatment in patients with endometrial thickness less than $7 \mathrm{~mm}$. Reprod Biomed Online. 2009;18:79-84.

6. Levi AJ, Drews MR, bergh PA, Miller BT, Scott RT Jr. Controlled ovarian hyperstimulation does not adversely affect endometrial receptivity in in vitro fertilization cycles. Fertil Steril. 2001;76:670-4.

7. Orvieto R, Meltzer S, Rabinson J, Zohav E, Anteby EY, Nahum R. GnRH agonist versus GnRH antagonist in ovarian stimulation: the role of endometrial receptivity. Fertil Steril. 2008;90:12946.

8. McWilliams GD, Frattarelli JL. Changes in measured endometrial thickness predict in vitro fertilization success. Fertil Steril. 2007;88:74-81.

9. Hernandez ER. Embryo implantation and GnRHantagonists: embryo implantation: the Rubicon for GnRH antagonists. Hum Reprod. 2000;15:1211-6.

10. Singh N, Bahadur A, Mittal S, Malhotra N, Bhatta A. Predictive value of endometrial thickness, pattern and sub-endometrial blood flows on the day of hCG by 2D Doppler in in-vitro fertilization cycles: a prospective clinical study from a tertiary care unit. J Hum Reprod Sci. 2011;4(1):29-33.

11. Noyes RW, Hertig AT, Rock J. Dating the endometrium. Fertil Steril. 1950;1:3-35.

12. Evans GE, Phillipson GT, Sin IL, Frampton CM, Kirker JA, Bigby SM, et al. Gene expression confirms a potentially receptive endometrium identified by histology in infertile women. Hum Reprod. 2012;27(9):2747-55.

13. Jinno M, Ozaki T, Iwashita M, Nakamura Y, kudo A, Hirano H. Measurement of endometrial tissue blood flow: a novel way to assess uterine receptivity for implantation. Fertil Steril. 2001;76:1168-74.

14. Al-Inany HG, Abou-Setta AM, Aboulghar M. Gonadotropin-releasing hormone antagonists for assisted conception. Cochrane Database Syst Rev. 2006;3:CD001750.

Cite this article as: Rai R, Ling YS, Yong TT, Keat AT, Rajesh H. Analysis of endometrial blood flow with color Doppler energy in predicting outcome in GnRH antagonist down regulated ICSI/IVF cycles: a prospective cohort study setting. Int J Reprod Contracept Obstet Gynecol 2017;6:4248-51. 